\title{
INVESTIGATION OF THE METHOD OF DYNAMIC MICROWAVE POWER REDISTRIBUTION IN A RESONATOR-TYPE PLASMATRON
}

\author{
S. Madveika, S. Bordusau*, A. Zemlyakov, M. Lushakova \\ Belarusian State University of Informatics and Radioelectronics, P.Brovki 6, 220013 Minsk, Belarus \\ * bordusov@bsuir.by
}

\begin{abstract}
The investigation results of a dynamic microwave power $f_{\text {microwave }}=2.45 \pm 0.05 \mathrm{GHz}$ redistribution in a $9000 \mathrm{~cm}^{3}$ reaction-discharge chamber of a microwave resonator-type plasmatron are presented. In order to redistribute the microwave power, a rotating metallic four-blade L-form dissector placed above the reaction-discharge chamber was used. The microwave power in the local points at the axis of the chamber with plasma and without it was measured applying the "active probe" method. During the experiments the chamber contained silicon wafers. Periodical interchange of maximum and minimum microwave power values along the chamber axis was established experimentally. Note, when the dissector was rotating, the range of maximum and minimum "active probe" values dispersion decreased. It has been established that during the dissector rotation the microwave power in the local discharge areas changes with periodic repetition every quarter of revolution.
\end{abstract}

Keywords: microwave plasma, microwave power, redistribution, dissector.

\section{Introduction}

The development of microwave gas discharge devices for technological purposes is moving in the direction of developing microwave plasmatrons with big plasma volume. It is connected with the necessity to treat semiconductor wafers of 200 and $300 \mathrm{~mm}$ diameter and the need to treat groups of wafers of smaller diameter at the operations of plasma cleaning, substrate surface activation, radical and plasma etching, plasma-chemical deposition of thin films, etc. [1-8]. Such technological plasma-chemical installations are developed on the base of microwave cavity resonators with discharge vacuum chambers located in the center [9-12].

Reasons for using the resonator systems are the next: they provide a high level of power dissipation in plasma; resonator is a well matched connector of microwave power feeder with plasma because its impedance changes insignificantly with the change of plasma impedance [13]; resonator preserves sufficiently high Q-factor even with the presence of plasma in it [14].

It is known [2] that the rates of plasma processes (ionization, dissociation, etc.) in microwave discharge plasma significantly correlate with the distribution of electromagnetic field in plasma. The concentration of active plasma particles, in its turn, is connected with the rate of plasma treatment processes of materials [9]. Depending on the process active plasma particles may be ions, radicals, atoms or etc.

In [15] it was shown that microwave power and spatial electric conductivity distributions in the resonator-type plasmatron's discharge chamber is unsteady when the values of the specific power applied to the microwave discharge are in the range $W_{M W}=0.06-0.08 \mathrm{~mW} / \mathrm{cm}^{3}$. This is due to the for- mation of standing waves with a certain structure in the resonator [16].

There are some technical methods $[2,12,17]$ allowing to change the microwave power distribution in a resonator. The simplest one is the use of a rotating metallic dissector. Such method of a dynamic microwave power redistribution does not require significant modification of the existing microwave plasmatrons' construction. As per today, there is no information in the scientific literature on the results of investigation of dissector's influence on the redistribution of microwave power inside and outside of the plasma volume in resonator-type plasmatrons. Thus, such research is of a scientific and practical interest as it may be useful for developing new microwave plasma systems or upgrading the existing ones.

\section{Experimental apparatus and procedures}

The research of the effect of a dynamic microwave power redistribution in a microwave discharge plasma was carried out with the help of a small-size resonatortype microwave plasma installation. The principle of operation of the vacuum-plasma system under study is based on the transmission of microwaves' energy from microwave magnetron $\mathrm{M}-105\left(f_{\text {microwave }}=\right.$ $2.45 \pm 0.05 \mathrm{GHz}$ ) into a vacuumed reaction-discharge chamber where a low-temperature gas microwave discharge is excited under the influence of the microwave electromagnetic field.

The discharge chamber is made of a quartz pipe, $\varnothing 20 \mathrm{~cm}$ and $34 \mathrm{~cm}$ long, with a volume about $9000 \mathrm{~cm}^{3}$, placed in the center of a $40 \times 40 \times 30 \mathrm{~cm}^{3}$ resonator (Fig. 1). The electromagnetic waves from the microwave magnetron are inlet into the resonator 


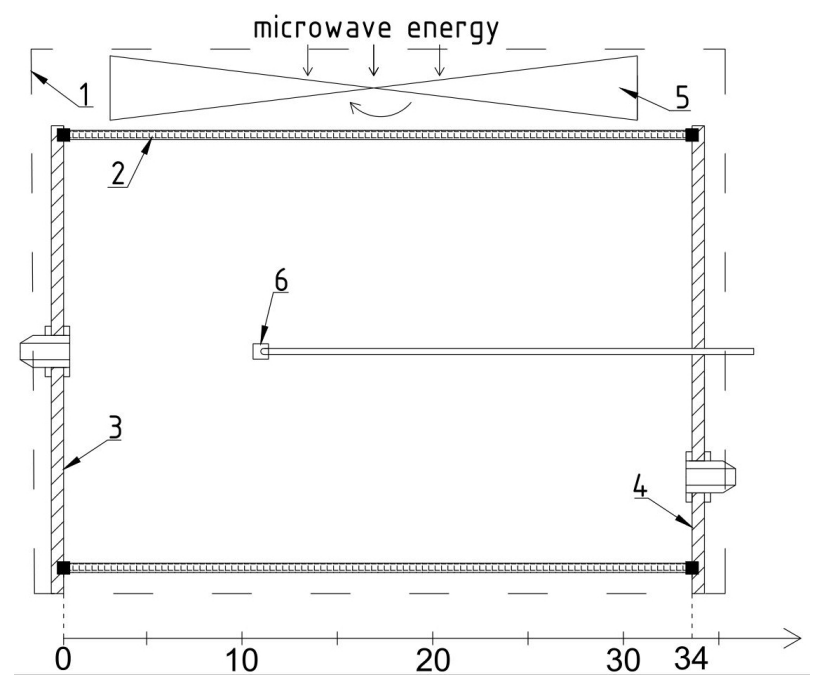

Figure 1. Reaction-discharge system of the microwave plasma installation:

1 - resonator; 2 - discharge chamber; 3 - front cover; 4 - back wall; 5 - dissector; 6 - "active probe"

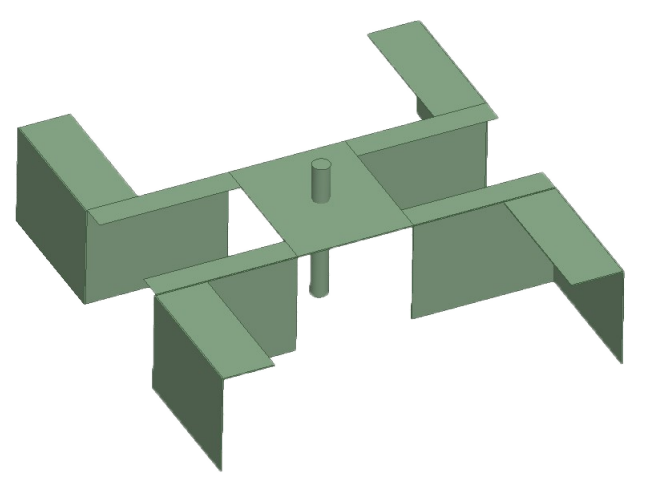

Figure 2. L-form dissector

through a rectangular hole in the resonator's top wall. An L-form metallic dissector is fixed on the resonator's top wall (Fig. 2). The dissector was placed in a position that allowed its blades to pass under the hole while rotating.

The microwave power in local points on the discharge chamber's axis with plasma and without plasma was measured using the "active probe" method [15]. The readings of the "active probe" were registered with a microwave power meter.

The character of plasma luminescence was controlled by registering the integral optical luminescence from the microwave discharge. The optical luminescence was sensed by an optical fiber, placed perpendicularly to the discharge chamber's axis, and was passed to the photoelectron multiplier. The photoelectron multiplier was connected to a digital oscilloscope.

During the experiments, there were 6 silicon wafers in the discharge chamber. The wafers of $\varnothing 100 \mathrm{~mm}$ were evenly placed in two lines along the chamber, 3 pcs

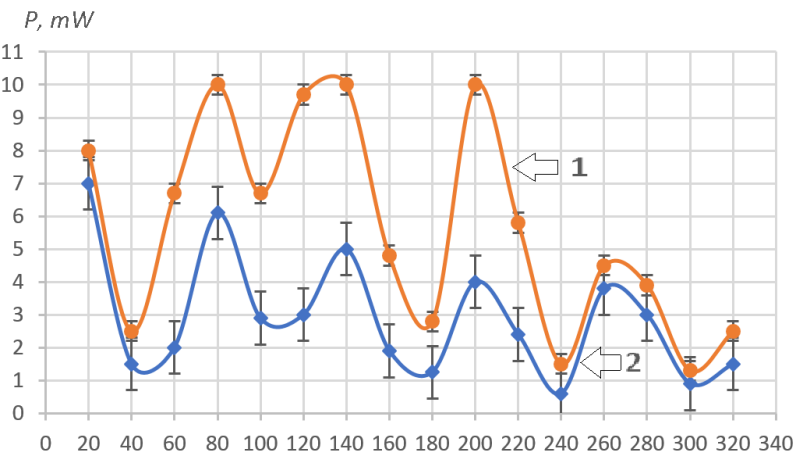

1, $\mathrm{mm}$

Figure 3. Readings of the active probe along the axis of the discharge chamber without plasma:

1 - without a dissector; 2 - with a moving dissector

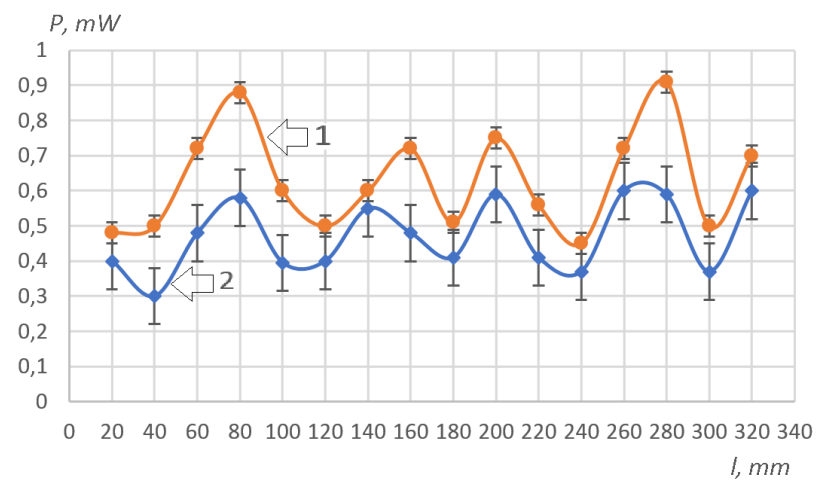

Figure 4. Readings of the active probe along the axis of the discharge chamber with plasma:

1 - without a dissector; 2 - with a moving dissector

from each side of the probe. The distance between the wafer pairs was $60 \mathrm{~mm}$.

$\mathrm{O}_{2}$ was used as a plasma-forming gas. The operating pressure in the chamber was $130 \mathrm{~Pa}$.

\section{Results and discussion}

Figure 3 shows the microwave power meter's readings when the "active probe" was moving along the axis of the discharge chamber without plasma. A point near the front chamber's cover was taken as the beginning of reading the distance along the discharge chamber axis.

It was established that when using a rotating metal dissector the range of microwave power values measured by the "active probe" along the axis of the discharge chamber decreases from $1.5-10 \mathrm{~mW}$ to $0.5-$ $7 \mathrm{~mW}$. This indicates a more uniform microwave power distribution in the discharge chamber volume.

Figure 4 shows the microwave power meter's readings for the case when the "active probe" was moved along the axis of the discharge chamber with plasma.

The presented data show a significant space irregularity decrease of the microwave power values in the discharge chamber's volume measured by the "active probe" when plasma is present and the dissector is moving. The maximum and minimum microwave 


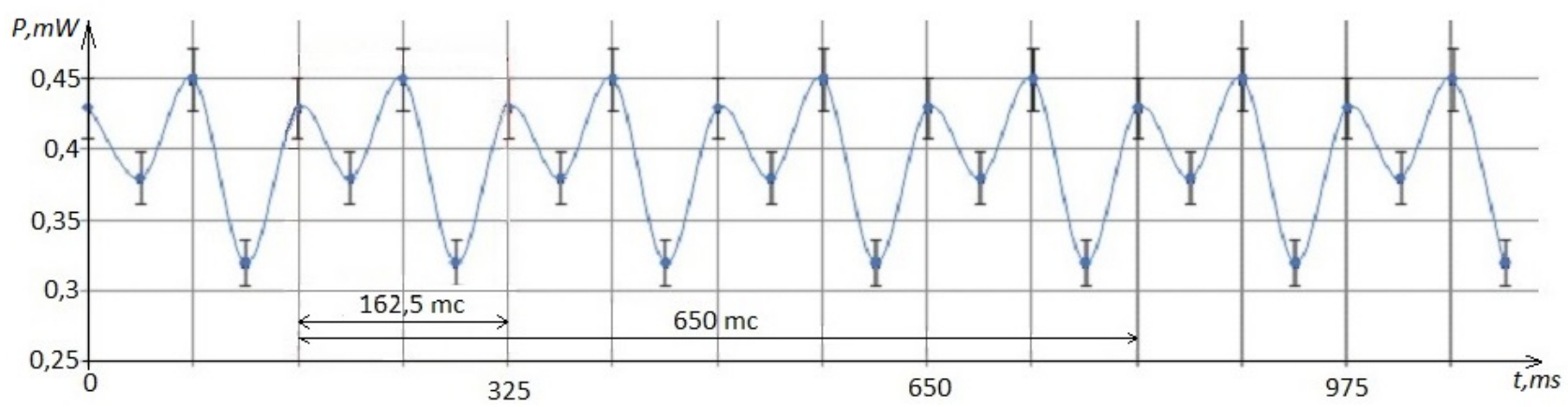

Figure 5. Changing of the "active probe" readings in the local area of the microwave discharge during the rotation of the dissector
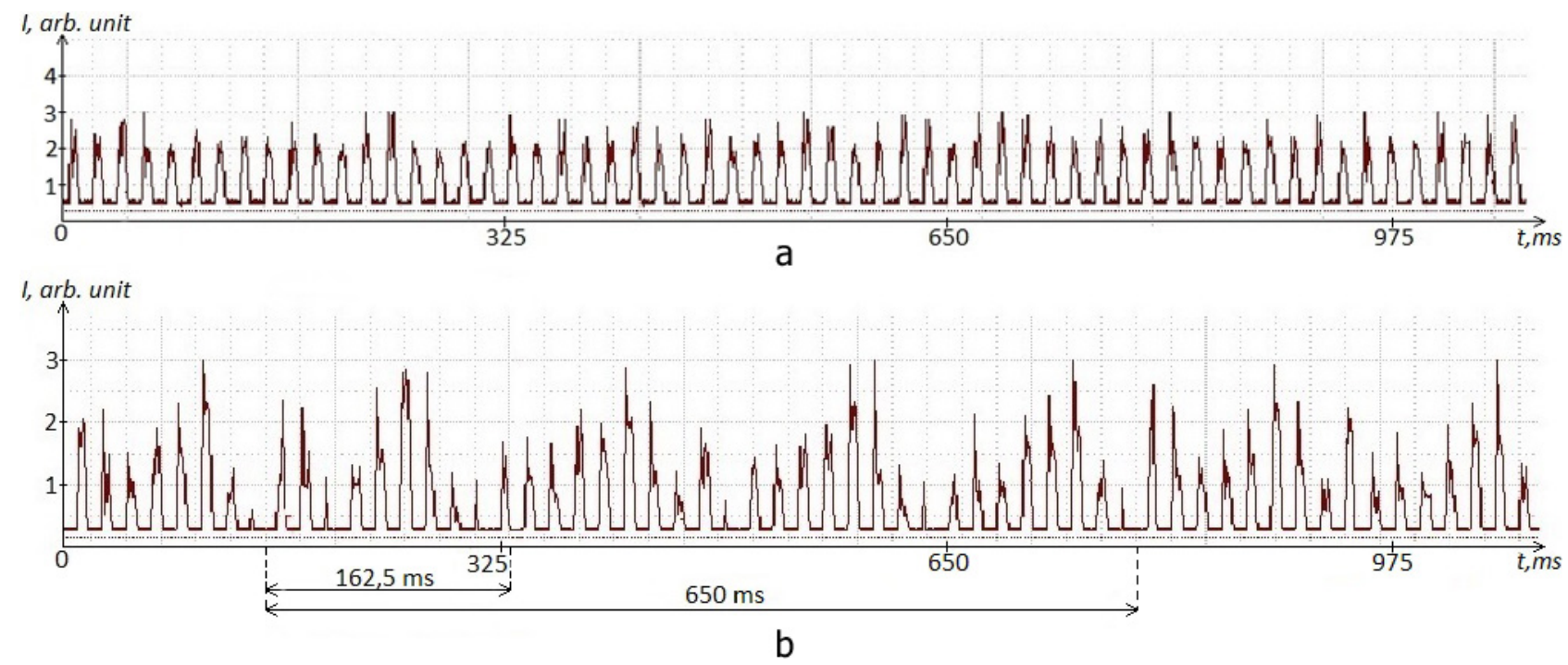

Figure 6. The registered oscillograms of integrated optical plasma luminescence signals from the local region of the microwave discharge:

$a$ - without a dissector; $b$ - with a moving dissector

power values in this case are $0.3 \mathrm{~mW}$ and $0.6 \mathrm{~mW}$ respectively.

The obtained results may be explained in the following way. When the dissector is moving, the conditions of excitation for various types of waves change depending on the blades position with respect to the hole of inletting the microwave power into the resonator. Because of this, the structure of the field in the resonator is permanently changing. It results in the decrease of irregularity of the electromagnetic power distribution in the microwave plasmatron's discharge chamber. The registered periodicity of maximum and minimum values in both series of experiments corresponds to about a half length of the electromagnetic wave $\left(f_{\text {microwave }}=2.45 \pm 0.05 \mathrm{GHz}\right)$ exciting the plasma.

It was found out that during the rotation of the dissector, the value of the microwave power in the local discharge areas changes with periodic repetition every quarter of its revolution (Fig. 5) (the time of a complete dissector's revolution is $650 \mathrm{msec}$ ).

Using a photoelectron multiplier, the changes of plasma luminescence intensity from different discharge chamber areas was studied. As an example, Figure 6 shows oscillograms of integral optical plasma luminescence signals from a local area when the dissector is absent (a) and in case it moves (b).

As it can be seen from Figure 6, the intensity of the plasma luminescence from the local region varies with periodic repetition every quarter of a turn of the dissector. This correlates with the data presented in Figure 5.

The obtained results indicate that during the dissector rotation a spatial redistribution of the microwave power in the volume of the discharge chamber takes place. This contributes to a decrease in the spatial inhomogeneity of the plasma in the microwave discharge chamber of the resonator-type plasmatron.

\section{Conclusions}

The performed investigation showed that, when the dissector is rotating, the microwave power is redistributed within the volume of the chamber of the microwave resonator-type plasmatron. The maximum and minimum values of the "active probe's" readings 
along the discharge chamber's axis decrease. It has been established that, when the dissector is rotating, the microwave power in the local areas of the discharge changes periodically every quarter of its revolution.

The obtained results can be used in designing new and upgrading of existing technological equipment for microwave plasma-chemical treatment of materials and electronic devices.

\section{Acknowledgements}

This research has been supported by the Belarusian State Program of Scientific Research "Physical materials science, new materials and technologies", assignment 3.5.02.

\section{References}

[1] M. Moisan and J. Pelletier. Microwave Excited Plasmas. Amsterdam: Elsevier, 1992.

[2] S. V. Bordusov. Microwave plasma technologies in the production of electronic devices. Minsk: Bestprint, 2002.

[3] M. Moisan and J. Pelletier. Physics of Collisional Plasma. Introduction to High-Frequency Discharges. Dordrecht: Springer, 2012.

[4] C. Boisse-Laporte and J. Marec, editors. Microwave discharges: fundamentals and applications Proc. 3rd Int. Workshop (Fontevraud, France, 20-25 April 1997). France: EDP Sciences, 1998.

[5] A. Ohl, editor. Microwave discharges: fundamentals and applications Proc. 5th Int. Workshop (Greifswald, Germany, 8-12 July 2003). Greifswald: INP, 2003.

[6] M. Kando and M. Nagatsu, editors. Microwave discharges: fundamentals and applications Proc. 7th Int. Workshop (Hamamatsu, Japan, 22-27 September 2009). Japan, 2009.

[7] Y. A. Lebedev, editor. Microwave discharges: fundamentals and applications Proc. 8th Int. Workshop (Zvenigorod, Russia, 10-14 September 2012). Moscow: Yanus-K, 2012.
[8] Y. A. Lebedev. Microwave discharges at low pressures and peculiarities of the processes in strongly non-uniform plasma. Plasma Sources Science and Technology, 24(5):053001, 2015. doi:10.1088/0963-0252/24/5/053001.

[9] T. Nakano and S. Samukawa. The correlation between an electric field and the metastable chlorine ion density distributions in an ultrahigh-frequency plasma. Japanese Journal of Applied Physics, 37(5R):2686-2692, 1998. doi:10.1143/JJAP. 37.2686.

[10] Plasmatic Systems. Plasma-preen plasma cleaning/etching system. URL: http://www.plasmapreen.com.

[11] Terra Universal. Plasma-preen cleaner/etcher (patent pending). URL: https://www.terrauniversal.com/ cleaning-systems/plasma-preen-cleaners .php.

[12] A. I. Kuzmichev. Ion plasma sources based on a microwave oven. Instruments and Experimental Techniques, 37(05):176-180, 1994.

[13] V. M. Batenin, I. I. Klimovsky, G. V. Lysov, and V. N. Troizky. Microwave Plasma Generators: Physics, Engineering, Applications. Moscow: Energoatomizdat, 1988.

[14] S. I. Madveika and S. V. Bordusau. Theoretical analysis of low vacuum microwave discharge exciting and maintaining conditions in resonator type plasmatron. Plasma Physics and Technology, 2(2):155-158, 2015.

[15] S. V. Bordusau, S. I. Madveika, and A. P. Dostanko. Investigation of microwave energy distribution character in a resonator type plasmatron. Plasma Physics and Technology, 3(3):122-125, 2016.

[16] E. Okress. Microwave power engineering. Number 1 in Electrical science series. Academic Press, 1968.

[17] J. Doehler. Large area microwave plasma apparatus with adaptable applicator, April 3 2001. US Patent 6.209.482. URL:

https://encrypted.google.com/patents/US6209482. 